\title{
DISTRIBUTION OF THE ANGLE OF ROTATION FOR PLANE RANDOM WALKS
}

\author{
F.W. WIEGEL
}

Center for Theoretical Studies, Indian Institute of Science, Bangalore 560012, India and Center of Theoretical Physics, Twente University of Technology, P.O. Box 217, Enschede 7500 AE,

The Netherlands

Received 4 June 1984

\begin{abstract}
We study the probability distribution of the angle by which the tangent to the trajectory rotates in the course of a plane random walk. It is shown that the determination of this distribution function can be reduced to an integral equation, which can be rigorously transformed into a differential equation of Hill's type. We derive the asymptotic distribution for very long walks.
\end{abstract}

\section{Introduction}

Although random walks have now been studied for about one century (cf. the bibliographical notes at the end of ref. 1), some of their most elementary properties have still not been considered in the literature. This paper is devoted to one such property: the total angle by which the tangent to the trajectory rotates in the course of a plane random walk.

If the random walk consists of $n$ steps, each of length $l$, the total angle by which the tangent to the walk has rotated at the end of the $n$th step will be denoted by $\phi$. Our problem consists of calculating the probability density $p_{n}(x, y, \phi)$ that this walk, which started at a point with Cartesian coordinates $x=0, y=0$, will reach $x, y$ with a "total angle of rotation" equal to $\phi$.

To the author's knowledge this problem has never been mentioned in the literature, much less solved exactly for walks of any length. Yet it is relevant to the combinatorial solution of the two-dimensional Ising model and to a recent attempt to make progress towards an analytical solution of the two-dimensional self-avoiding random walk problem (ref. 2 and papers quoted there). In view of these important consequences a more detailed study of this problem seems justified. In section 2 we express the probability distribution in terms of the eigenvalues of an integral equation. In section 3 it is shown that this integral equation can be rigorously transformed into a differential equation of Hill's type. In section 4 the asymptotic form of the distribution for very long walks is extracted from the differential equation. 


\section{Integral equation}

The basic geometry is indicated in fig. 1 for a random flight $\left(\boldsymbol{\theta}, \boldsymbol{r}_{1}, \boldsymbol{r}_{2}, \boldsymbol{r}_{3}, \boldsymbol{r}_{4}\right)$ consisting of four steps of equal length $l$. The direction of step $j$ with respect to step $j-1$ is denoted by $\alpha_{j}$,

$$
-\pi<\alpha_{j}<+\pi .
$$

The first angle $\alpha_{1}$ is measured with respect to the positive real $x$-axis. In our model we assume that the different values of $\alpha_{j}$ can occur with a constant probability density $(2 \pi)^{-1}$. The total angle $\phi_{j}$ by which the tangent to the random walk has rotated at the end of step $j$ is given by the algebraic sum

$$
\phi_{j}=\alpha_{1}+\alpha_{2}+\cdots+\alpha_{j} .
$$

Note that

$$
-\pi j<\phi_{j}<+\pi j
$$

After $n$ steps the walk is at a position $r_{n} \equiv\left(x_{n}, y_{n}\right)$ with

$$
\begin{aligned}
& x_{n}=l \sum_{j=1}^{n} \cos \phi_{j}, \\
& y_{n}=l \sum_{j=1}^{n} \sin \phi_{j} .
\end{aligned}
$$

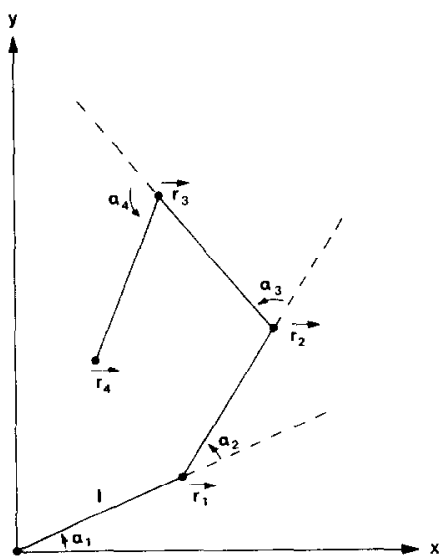

Fig. 1. Basic geometry for plane random walks. Explanation in section 2. 
Hence the probability density $p_{n}(x, y, \phi)$ that the walk will have reached the point $(x, y)$, after $n$ steps, with a total angle of rotation $\phi$ is given by the integral

$$
\begin{aligned}
p_{n}(x, y, \phi)= & (2 \pi)^{-n} \int_{-\pi}^{+\pi} \mathrm{d} \alpha_{1} \int_{-\pi}^{+\pi} \mathrm{d} \alpha_{2} \cdots \int_{-\pi}^{+\pi} \mathrm{d} \alpha_{n} \delta\left(x-l \sum_{j=1}^{n} \cos \phi_{j}\right) \\
& \times \delta\left(y-l \sum_{j=1}^{n} \sin \phi_{j}\right) \delta\left(\phi-\phi_{n}\right) .
\end{aligned}
$$

Now, from relation (2.2) between the $\alpha$ and the $\phi$ variables one sees that

$$
\phi_{j}=\phi_{j-1}+\alpha_{j}
$$

and hence (2.5) can also be written in the form

$$
\begin{aligned}
p_{n}(x, y, \phi)= & (2 \pi)^{-n} \int_{-\pi}^{+\pi} \mathrm{d} \phi_{1} \int_{\phi_{1}-\pi}^{\phi_{1}+\pi} \mathrm{d} \phi_{2} \cdots \int_{\phi_{n-1}-\pi}^{\phi_{n-1}+\pi} \mathrm{d} \phi_{n} \delta\left(x-l \sum_{j=1}^{n} \cos \phi_{j}\right) \\
& \times \delta\left(y-l \sum_{j=1}^{n} \sin \phi_{j}\right) \delta\left(\phi-\phi_{n}\right)
\end{aligned}
$$

in which the $\alpha$-variables do not occur anymore.

For the Fourier transform

$$
q_{n}\left(k_{1}, k_{2}, \mu\right)=\iiint p_{n}(x, y, \phi) \exp \left\{-\mathrm{i}\left(k_{1} x+k_{2} y+\mu \phi\right)\right\} \mathrm{d} x \mathrm{~d} y \mathrm{~d} \phi,
$$

one finds the multiple integral

$$
\begin{aligned}
q_{n}\left(k_{1}, k_{2}, \mu\right)= & (2 \pi)^{-n} \int_{-\pi}^{+\pi} \mathrm{d} \phi_{1} \int_{\phi_{1}-\pi}^{\phi_{1}+\pi} \mathrm{d} \phi_{2} \cdots \int_{\phi_{n-1}-\pi}^{\phi_{n-1}+\pi} \mathrm{d} \phi_{n} \\
& \times \exp \left(-\mathrm{i} k_{1} l \sum_{j=1}^{n} \cos \phi_{j}-\mathrm{i} k_{2} l \sum_{j=1}^{n} \sin \phi_{j}-\mathrm{i} \mu \phi_{n}\right) .
\end{aligned}
$$

In order to calculate these multiple integrals we define a sequence of $n+1$ 
functions $F_{0}, F_{1}, \ldots, F_{n}$ with arguments $\phi_{n}, \phi_{n-1}, \ldots, \phi_{0}$ by the recurrent relation

$$
\begin{aligned}
& F_{0}\left(\phi_{n}\right)=\exp \left(-\mathrm{i} \mu \phi_{n}\right) \\
& F_{j}\left(\phi_{n-j}\right)=\frac{1}{2 \pi} \int_{\phi_{n-j^{-\pi}}}^{\phi_{n-j+\pi}} F_{j-1}\left(\phi_{n-j+1}\right) \exp \left(-\mathrm{i} k_{1} l \cos \phi_{n-j+1}-\mathrm{i} k_{2} l \sin \phi_{n-j+1}\right) \mathrm{d} \phi_{n-j+1} \\
& \quad(j=1,2, \ldots, n) .
\end{aligned}
$$

In terms of these functions the Fourier transform (2.9) of the probability distribution is given by

$$
q_{n}\left(k_{1}, k_{2}, \mu\right)=F_{n}(0) \text {. }
$$

The recurrent relation (2.11) suggests the use of an eigenfunction expansion in the following way. First, solve the eigenvalue problem

$$
\frac{1}{2 \pi} \int_{\phi-\pi}^{\phi+\pi} \exp \left(-\mathrm{i} k_{1} l \cos \phi^{\prime}-\mathrm{i} k_{2} l \sin \phi^{\prime}\right) f_{s}\left(\phi^{\prime}\right) \mathrm{d} \phi^{\prime}=\lambda_{s} f_{s}(\phi) \text {. }
$$

Then, if the recurrent scheme starts with

$$
F_{0}(\phi)=\exp (-\mathrm{i} \mu \phi)=\sum_{s} a_{s} f_{s}(\phi)
$$

the solution is

$$
F_{n}(\phi)=\sum_{s} a_{s} \lambda_{s}^{n} f_{s}(\phi)
$$

Substitution of (2.15) into (2.12) and of (2.12) into (2.8) in principle determines the solution of our problem.

\section{The differential equation}

In order to make some further progress we now consider those eigenfunctions of the integral equation which have the special form

$$
f_{s}(\phi)=a_{s}(\phi) \exp (\mathrm{is} \phi),
$$


where $-\infty<s<+\infty$ and where $a_{s}(\phi)$ has period $2 \pi$,

$$
a_{s}(\phi+2 \pi)=a_{s}(\phi)
$$

It will turn out that these already form a complete set of functions. Substitution of (3.1) into (2.13) gives

$$
\frac{1}{2 \pi} \int_{\phi-\pi}^{\phi+\pi} \exp \left\{-\mathrm{i} k l \cos \left(\phi^{\prime}-\gamma\right)+\mathrm{i} s\left(\phi^{\prime}-\phi\right)\right\} a_{s}\left(\phi^{\prime}\right) \mathrm{d} \phi^{\prime}=\lambda_{s} a_{s}(\phi)
$$

where we wrote

$$
k_{1}=k \cos \gamma, \quad k_{2}=k \sin \gamma, \quad-\pi<\gamma<+\pi
$$

It is perhaps remarkable that this integral equation can be transformed into a differential equation of the second order in the following way: (a) Differentiate (3.3) once with respect to $\phi$ and use (3.2) to obtain the arguments $\phi$ and $\phi+\pi$ throughout the resulting equation for $\mathrm{d} a_{s} / \mathrm{d} \phi$; (b) Differentiate again with respect to $\phi$ and use the previously found equation for $\mathrm{d} a_{s} / \mathrm{d} \phi$ to simplify the result. This gives

$$
\frac{\mathrm{d}^{2} a_{s}}{\mathrm{~d} \phi^{2}}+\mathrm{i}\{2 s+k l \sin (\phi-\gamma)\} \frac{\mathrm{d} a_{s}}{\mathrm{~d} \phi}-\left\{s k l \sin (\phi-\gamma)+s^{2}-\left(\frac{\sin \pi s}{\pi \lambda_{s}}\right)^{2}\right\} a_{s}=0
$$

A change of variables brings this into a familiar form. Indeed, introducing the independent variable $z$ by

$$
\phi=\gamma+2 z
$$

and writing

$$
a_{s}(\phi)=\exp \left\{-\mathrm{i} s \phi+\frac{1}{2} \mathrm{i} k l \cos (\phi-\gamma)\right\} u(z)
$$

one finds

$$
\frac{\mathrm{d}^{2} u}{\mathrm{~d} z^{2}}+J(z) u=0
$$


with

$$
\begin{aligned}
J(z) & =\theta_{0}+2 \theta_{1} \cos 2 z+2 \theta_{2} \cos 4 z \\
\theta_{0} & =4\left(\frac{\sin \pi s}{\pi \lambda_{s}}\right)^{2}+\frac{1}{2} k^{2} l^{2} \\
2 \theta_{1} & =-2 \mathrm{i} k l \\
2 \theta_{2} & =-\frac{1}{2} k^{2} l^{2}
\end{aligned}
$$

in the notation of p. 414 of ref. 3. This is a special case of Hill's equation, called the Whittaker-Hill equation by Magnus and Winkler ${ }^{4}$ ). We want the solutions of the form

$$
u(z)=\exp (2 \mathrm{i} s z) F_{s}(z),
$$

where $F_{s}(z)$ has the period $\pi$,

$$
F_{s}(z+\pi)=F_{s}(z) .
$$

In the general theory of Hill's equation ${ }^{3,4}$ ) it is shown that such solutions exist provided $\theta_{0}, \theta_{1}, \theta_{2}$ and $s$ are related by the equation

$$
\sin ^{2}(\pi s)=\Delta(0) \sin ^{2}\left(\frac{1}{2} \pi \vee \theta_{0}\right),
$$

where $\Delta(0)$ denotes Hill's infinite determinant

$$
\Delta(0)=\left|\begin{array}{ccccc}
1 & \frac{-\theta_{1}}{4^{2}-\theta_{0}} & \frac{-\theta_{2}}{4^{2}-\theta_{0}} & 0 & 0 \\
\frac{-\theta_{1}}{2^{2}-\theta_{0}} & 1 & \frac{-\theta_{1}}{2^{2}-\theta_{0}} & \frac{-\theta_{2}}{2^{2}-\theta_{0}} & 0 \\
\frac{-\theta_{2}}{0^{2}-\theta_{0}} & \frac{-\theta_{1}}{0^{2}-\theta_{0}} & 1 & \frac{-\theta_{1}}{0^{2}-\theta_{0}} & \frac{-\theta_{2}}{0^{2}-\theta_{0}} \\
0 & \frac{-\theta_{2}}{2^{2}-\theta_{0}} & \frac{-\theta_{1}}{2^{2}-\theta_{0}} & 1 & \frac{-\theta_{1}}{2^{2}-\theta_{0}} \\
0 & 0 & \frac{-\theta_{2}}{4^{2}-\theta_{0}} & \frac{-\theta_{1}}{4^{2}-\theta_{0}} & 1
\end{array}\right|
$$

It shown there also that these solutions form a complete set of functions. 
Hence the Ansatz (3.1) has given rise to a complete set of eigenfunctions of the integral equation (2.13), which in a formal way solves the problem of determining the distribution of the angle of rotation for plane random walks.

\section{Asymptotic form of the distribution}

From a practical point of view the explicit determination of the distribution for small values of $n$ is still very complicated. We shall, therefore, limit ourselves here to the asymptotic case $n \gg 1$. In this case the typical values are $r / l \sim \mathcal{O}\left(n^{1 / 2}\right)$ and $\phi \sim \mathcal{O}\left(n^{1 / 2}\right)$, hence we are interested in $k l \sim \mathscr{O}\left(n^{-1 / 2}\right)$ and $s \sim \mathcal{O}\left(n^{-1 / 2}\right)$. The simplest way to proceed is to consider two special cases: (a) $k l=0$ and $s \ll 1$; (b) $s=0$ and $k l \ll 1$.

In the first case, $k l=0$, Hill's determinant (3.14) equals unity. Hence eq. (3.13) reads

$$
4 s^{2}=\theta_{0},
$$

which upon substitution of (3.10a) gives

$$
\lambda_{s}=\frac{\sin \pi s}{\pi s} \cong 1-\frac{\pi^{2}}{6} s^{2} \quad(k l=0, s \ll 1) .
$$

In the second case, $s=0$, the value of $\lambda_{0}$ as a function of $k l$ can most easily be derived from the eigenvalue equation $(2.13)$ by putting $f_{0}(\phi)=$ constant. This gives

$$
\lambda_{0}=\frac{1}{2 \pi} \int_{-\pi}^{+\pi} \exp (-\mathrm{i} k l \cos \phi) \mathrm{d} \phi=J_{0}(k l) \cong 1-\frac{1}{4} k^{2} l^{2} \quad(s=0, k l \ll 1) .
$$

The last two equations and the fact that $\lambda_{s}$ must be an even function of $s$ (because the replacement of $s$ by $-s$ does not change anything in (3.13)) implies that the behavior of the eigenvalues for small, non-zero values of $s$ and $k l$ must be of the form

$$
\begin{aligned}
\lambda_{s} & =1-\frac{\pi^{2}}{6} s^{2}-\frac{1}{4} k^{2} l^{2}+\cdots \\
& \cong \exp \left(-\frac{\pi^{2}}{6} s^{2}-\frac{1}{4} k^{2} l^{2}\right)
\end{aligned}
$$


Now recall the formula

$$
q_{n}\left(k_{1}, k_{2}, \mu\right)=\sum_{s} a_{s} \lambda_{s}^{n} f_{s}(0)
$$

For small values of $k l$ and arbitrary values of $s$ we can still use the $k l=0$ form of the eigenfunctions as an approximation

$$
f_{s}(\phi) \cong \exp (i s \phi) \quad(k l \ll 1),
$$

but as $\lambda_{s}$ enters into (4.5) to a large power we have to use (4.4) for the eigenvalue. Combining (2.10) and (2.14) into the continuous form

$$
\exp (-\mathrm{i} \mu \phi)=\int_{-\infty}^{+\infty} a_{s}(\mu) \exp (\mathrm{i} s \phi) \mathrm{d} s
$$

one finds

$$
a_{s}(\mu)=\delta(s+\mu)
$$

Substitution into (4.5) gives

$$
\begin{aligned}
q_{n}\left(k_{1}, k_{2}, \mu\right) & =\lambda_{-\mu}^{n} \\
& \cong \exp \left(-\frac{\pi^{2}}{6} n \mu^{2}-\frac{1}{4} n k^{2} l^{2}\right) .
\end{aligned}
$$

Taking the inverse Fourier transform of (2.8) the asymptotic form of the probability distribution is found to be given by

$$
\begin{aligned}
& p_{n}(x, y, \phi) \cong p_{n}^{(1)}(\phi) p_{n}^{(2)}(x, y) \quad(n \gg 1) \\
& p_{n}^{(1)}(\phi)=\left(\frac{2}{3} n \pi^{3}\right)^{-1 / 2} \exp \left(-\frac{3 \phi^{2}}{2 n \pi^{2}}\right) \\
& p_{n}^{(2)}(x, y)=\left(n \pi l^{2}\right)^{-1} \exp \left(-\frac{x^{2}+y^{2}}{n l^{2}}\right)
\end{aligned}
$$

This asymptotic formula simply expresses the fact that for $n \gg 1$ the distribution for the angle of rotation is statistically independent of the distribution of the final position of the walk. This was to be expected a priori and 
recovering this result merely serves as a consistency check on the formalism of sections 2 and 3.

\section{Acknowledgements}

The author is indepted to Prof. R. Rajaraman and Prof. K.P. Sinha for their hospitality at the Center for Theoretical Studies of the Indian Institute of Science during the fall of 1983.

\section{References}

1) S. Chandrasekhar, Rev. Mod. Phys. 15 (1943) 1.

2) F.W. Wiegel, Fluid Flow through Porous Macromolecular Systems, Lecture Notes in Physics 121 (Springer, Berlin, 1980), appendix.

3) E.J. Whittaker and G.N. Watson, A Course of Modern Analysis (Cambridge Univ. Press, Cambridge, 1927).

4) W. Magnus and S. Winkler, Hill's equation (Dover, New York, 1979), p. 106. 